\title{
Optimisation of Dynamic Vibration Absorbers to Minimise Kinetic Energy and Maximise Internal Power Dissipation
}

Michele Zilletti $^{\text {a }}$

Stephen J. Elliott ${ }^{\mathrm{a}}$

EmilianoRustighi $^{\mathrm{a}}$ mz@isvr.soton.ac.uk

sje@isvr.soton.ac.uk

E.Rustighi@soton.ac.uk

a Institute of Sound and Vibration Research, Highfield, Southampton SO171BJ, UK

Keywords: Tuneable Vibration absorber, Tuned mass damper, Power absorption,

\begin{abstract}
The tuning of a dynamic vibration absorber is considered such that either the kinetic energy of the host structure is minimised or the power dissipation within the absorber is maximised. If the host structure is approximated as a damped single degree of freedom, the optimal values for the ratio of the absorber's natural frequency to the host structure and the optimal damping ratio of the absorber are shown to be the same whether the kinetic energy of the host structure is minimised or the power dissipation of the absorber is maximised. It is also demonstrated that the total power input into the system does not depend on the two parameters but only on the host structure's mass.
\end{abstract}

\section{INTRODUCTION}

Dynamic vibration absorbers are single degree of freedom systems that are attached to a host structure to control its motion [1]. Such devices were originally patented in 1911 [2]. They are widely used to control the vibration of civil [3-5] and aerospace [6-8] structures and can operate in different ways depending on the application. One way of operating such a device aims at suppressing the vibration only at a particular forcing frequency, in which case the devices natural frequency is tuned to this excitation frequency. The damping of the device should also be as low as possible in this case, so that it presents the greatest impedance to the host structure at the operating frequency. The device is then often known as a "vibration 
neutraliser", and considerable ingenuity has been put into tuning the natural frequency of the device to track variations in the excitation frequency $[4,6,9,10]$.

Alternatively the device can be used to attenuate the vibration due to a particular mode of the structure over a range of frequencies, when it is sometimes referred to as a "tuned mass damper" (TMD) or "dynamic vibration absorber" (DVA). The optimum tuning of the natural frequency and damping ratio of the device then become less obvious and depend on exactly how the optimisation criterion is defined. The selected mode of the host structure is generally modelled as a single degree of freedom system for this optimisation, often without any inherent damping.

A survey of tuning criterion for dynamic vibration absorbers when used as tuned mass dampers has been presented by Asami [11]. The original optimisation criterion used by Omondroyd and Den Hartog 1928 [12] fixed the natural frequency of the DVA such that the magnitude of the displacement was equal at the two peaks in the coupled displacement response after the device has been attached, and the damping ratio of the DVA was chosen such that the derivate at the two peaks was zero. This is also known as min-max or $\mathrm{H}_{\infty}$ optimisation. The solution of the optimisation criterion proposed by Omondroyd and Den Hartog is a well known result and has been widely used, although it is an approximated solution. The exact solution for the $\mathrm{H}_{\infty}$ optimisation was found by Nishihara and Asami [13] in 2002. Another optimisation criterion would be to minimise the mean square displacement of the host structure when excited by a random force of uniform power spectral density, as first proposed by Crandall and Mark in 1963 [14] and also now known as $\mathrm{H}_{2}$ optimisation. A third possibility is to adjust the natural frequency and damping of the device such that the poles of the overall system have the greatest negative value, so that the transient response decays as quickly as possible. Asamiet al.[11] attribute this result in Table 1 to Yamaguchi in 1988 [15], although the same criterion was also considered by Miller and Crawley in 1985 [16]. Krenk in 2005 [17] proposed a further method to tune the parameters of a DVA. He tuned the frequency ratio of the two decoupled oscillators using the same criterion proposed by Omondroyd and den Hartog [12] and proposed a new criterion for the optimal damping ratio. The damping ratio was chosen by simultaneously minimising the displacement of the main mass and the relative displacement of the two masses calculated at the natural frequency of the system when the damper was blocked. He also demonstrated that for the frequency tuning proposed by Omondroyd and den Hartog [12], the complex locus of the modal frequencies has a bifurcation point corresponding to the maximum 
damping of the two modes. For lower damping ratio the two modes have the same modal damping. Warburton in 1982 [18] proposed the minimisation of the frequency averaged kinetic energy of the host structure as a tuning criterion.

In this paper a further criterion on which to optimise a dynamic vibration absorber is considered, based on the maximisation of the power dissipated by the absorber. It is found for a damped host structure that the maximisation of the power absorbed by the damper corresponds to the minimisation of the kinetic energy of the host structure. It is also demonstrated that the power input into the system is independent of the damping ratio of the DVA and the natural frequency ratio of the two uncoupled system.

\section{MODELLING}

The dynamic vibration absorber is a passive vibration control device which is attached to a vibrating host structure often called the primary structure. A single mode of this structure is often modelled as a single degree of freedom primary system, which is shown with the DVA in Figure 1 where $m_{1}$ and $m_{2}$ are the masses, $k_{1}$ and $k_{2}$ are the stiffness values and $c_{1}$ and $c_{2}$ the mechanical damping values of the primary system and the DVA respectively. The primary system is subjected to a random excitation $f_{\mathrm{p}}$, which is assumed to have a flat power spectral density, $S_{\mathrm{f}}$, and $x_{1}$ and $x_{2}$ are the displacements of the masses $m_{1}$ and $m_{2}$.

The steady state response of the system can be expressed in terms of the five dimensionless parameters defined by:

$$
\begin{gathered}
\mu=m_{2} / m_{1} \\
\nu=\omega_{2} / \omega_{1} \\
\lambda=\omega / \omega_{1} \\
\zeta_{1}=c_{1} /\left(2 m_{1} \omega_{1}\right) \\
\zeta_{2}=c_{2} /\left(2 m_{2} \omega_{2}\right)
\end{gathered}
$$

where $\mu$ is the mass ratio, $v$ is the frequency ratio, $\lambda$ is the forced frequency ratio, $\zeta_{1}$ is the primary damping ratio and $\zeta_{2}$ is the secondary damping ratio. The natural frequency of the primary system, $\omega_{1}$, and the natural frequency of the DVA, $\omega_{2}$, are given by:

$$
\begin{aligned}
& \omega_{1}=\sqrt{k_{1} / m_{1}} \\
& \omega_{2}=\sqrt{k_{2} / m_{2}}
\end{aligned}
$$

As described in the previous section, several tuning strategies for the DVA natural frequency and its damping have been proposed, with the scope of reducing the vibration of the primary 
system. Table 1 summarises various type of optimisations when the primary system has vanishing small damping $\left(\zeta_{1} \ll 1\right)$.

Table 1: optimisation criteria of the dynamic vibration absorber on a SDOF system with vanishing small damping. $\zeta_{\text {opt }}$ is the optimal damping ratio of the DVA and $v_{\mathrm{opt}}$ is the optimal frequency ratio of the natural frequencies of the two uncoupled systems. $x_{\mathrm{st}}$ is the static deflection of the primary system. $\mathrm{E}[]$ indicates the expectation value and the dot indicates derivate with respect of time.

\begin{tabular}{|c|c|c|c|c|c|}
\hline & $\begin{array}{c}\text { Optimisation } \\
\text { criterion }\end{array}$ & Performance index & Objective & Proposed by: & Optimal parameters \\
\hline 1 & $\begin{array}{c}H_{\infty} \\
\text { Optimisation }\end{array}$ & $A_{1 \max }=\left|\frac{x_{1}}{x_{\mathrm{st}}}\right|_{\max }$ & $\begin{array}{l}\text { Minimise the } \\
\text { maximum } \\
\text { displacement of } \\
\text { the primary mass }\end{array}$ & $\begin{array}{c}\text { Ormondroyd } \\
\text { \& Den Hartog } \\
1928[12] \\
\text { Nishihara and } \\
\text { Asami[13] } \\
\end{array}$ & $\begin{array}{c}\zeta_{\mathrm{opt}}=\sqrt{\frac{3 \mu}{8(1+\mu)}} \\
v_{\mathrm{opt}}=\frac{1}{1+\mu}\end{array}$ \\
\hline 2 & $\begin{array}{c}\mathrm{H}_{2} \\
\text { Optimisation } \\
\text { of the mean } \\
\text { squared } \\
\text { displacement }\end{array}$ & $I_{1}=\frac{\mathrm{E}\left[x_{1}^{2}\right]}{S_{f} \omega_{1} / k_{1}^{2}}$ & $\begin{array}{l}\text { Minimise the total } \\
\text { displacement of } \\
\text { the primary mass } \\
\text { over all frequency }\end{array}$ & $\begin{array}{c}\text { Iwata } 1982 \\
\quad[19], \\
\text { Warburton } \\
1982[18]\end{array}$ & $\begin{array}{c}\zeta_{\mathrm{opt}}=\sqrt{\frac{\mu(4+3 \mu)}{8(1+\mu)(2+\mu)}} \\
v_{\mathrm{opt}}=\frac{1}{1+\mu} \sqrt{\frac{2+\mu}{2}}\end{array}$ \\
\hline 3 & $\begin{array}{c}\text { Stability } \\
\text { Maximisation }\end{array}$ & $\Lambda=-\max _{i}\left(\operatorname{Re}\left[s_{i}\right]\right)$ & $\begin{array}{l}\text { Minimise the } \\
\text { transient vibration } \\
\text { of the system }\end{array}$ & $\begin{array}{c}\text { Millers et } \\
\text { al.1985 [16] } \\
\text { Yamaguchi } \\
\text { 1988[15], }\end{array}$ & $\begin{aligned} \zeta_{\mathrm{opt}} & =\sqrt{\frac{\mu}{1+\mu}} \\
v_{\mathrm{opt}} & =\frac{1}{1+\mu}\end{aligned}$ \\
\hline 4 & $\begin{array}{c}H_{\infty} \\
\text { Minimisation } \\
\text { of relative } \\
\text { displacement }\end{array}$ & $\begin{array}{l}A_{1 \max }=\left|\frac{x_{1}}{x_{\mathrm{st}}}\right|_{\max } \\
A_{2 \max } \\
=\left|\frac{x_{1}-x_{2}}{x_{\mathrm{st}}}\right|_{\max }\end{array}$ & $\begin{array}{l}\text { Minimisation of } \\
\text { displacement of } \\
\text { the main mass and } \\
\text { relative } \\
\text { displacement }\end{array}$ & $\begin{array}{c}\text { Krenk } 2005 \\
\text { [17] }\end{array}$ & $\begin{array}{c}\zeta_{\mathrm{opt}}=\sqrt{\frac{\mu}{2(1+\mu)}} \\
v_{\mathrm{opt}}=\frac{1}{1+\mu}\end{array}$ \\
\hline 5 & $\begin{array}{c}\mathrm{H}_{2} \\
\text { Minimisation } \\
\text { of kinetic } \\
\text { energy }\end{array}$ & $I_{\mathrm{k}}=\frac{\mathrm{E}\left[\dot{x}_{1}^{2}\right]}{2 \pi S_{f} \omega_{1} / k_{1}}$ & $\begin{array}{l}\text { Minimise the total } \\
\text { kinetic energy of } \\
\text { the primary mass } \\
\text { over all } \\
\text { frequencies }\end{array}$ & $\begin{array}{c}\text { Warburton } \\
1982[18]\end{array}$ & $\begin{array}{c}\zeta_{\mathrm{opt}}=\frac{\sqrt{\mu}}{2} \\
v_{\mathrm{opt}}=\frac{1}{\sqrt{1+\mu}}\end{array}$ \\
\hline 6 & $\begin{array}{c}\mathrm{H}_{2} \\
\text { Maximisation } \\
\text { of the } \\
\text { absorbed } \\
\text { power }\end{array}$ & $I_{\mathrm{p} 2}=\frac{c_{2} \mathrm{E}\left[\left|\dot{x}_{1}-\dot{x}_{2}\right|^{2}\right]}{2 \pi S_{f} \omega_{1} / k_{1}}$ & $\begin{array}{c}\text { Minimise power } \\
\text { dissipated by the } \\
\text { absorber }\end{array}$ & This study ${ }^{1}$ & $\begin{array}{c}\zeta_{\mathrm{opt}} \approx \frac{\sqrt{\mu}}{2} \\
v_{\mathrm{opt}} \approx \frac{1}{\sqrt{1+\mu}}\end{array}$ \\
\hline
\end{tabular}

The mobility functions of the system, derived in appendix A, can be expressed in nondimensional form using the parameters given in equations (1) yielding to:

$$
\begin{aligned}
& \Gamma=\sqrt{k_{1} m_{1}} \mathrm{Y}_{11}(\mathrm{j} \lambda)=\frac{B_{0}+(\mathrm{j} \lambda) B_{1}+(\mathrm{j} \lambda)^{2} B_{2}+(\mathrm{j} \lambda)^{3} B_{3}}{A_{0}+(\mathrm{j} \lambda) A_{1}+(\mathrm{j} \lambda)^{2} A_{2}+(\mathrm{j} \lambda)^{3} A_{3}+(\mathrm{j} \lambda)^{4} A_{4}} \\
& \Theta=\sqrt{k_{1} m_{1}} \mathrm{Y}_{12}(\mathrm{j} \lambda)=\frac{C_{0}+(\mathrm{j} \lambda) C_{1}+(\mathrm{j} \lambda)^{2} C_{2}+(\mathrm{j} \lambda)^{3} C_{3}}{A_{0}+(\mathrm{j} \lambda) A_{1}+(\mathrm{j} \lambda)^{2} A_{2}+(\mathrm{j} \lambda)^{3} A_{3}+(\mathrm{j} \lambda)^{4} A_{4}}
\end{aligned}
$$

where

\footnotetext{
${ }^{1}$ This optimal values of $\zeta_{2}$ and $v$ are approximated and only valid if $\zeta_{1} \ll 1$ although the maximisation of the power absorbed is equivalent to the minimisation of the kinetic energy as tuning strategy for any value of $\zeta_{1} \neq 0$
} 


$$
\begin{array}{lll}
A_{0}=v^{2} & B_{0}=0 & C_{0}=0 \\
A_{1}=2 \zeta_{2} v+2 \zeta_{1} v^{2} & B_{1}=v^{2} & C_{1}=v^{2} \\
A_{2}=v^{2}+1+\mu v^{2}+4 \zeta_{1} \zeta_{2} v & B_{2}=2 \zeta_{2} v & C_{2}=2 \zeta_{2} v \\
A_{3}=2 \zeta_{2} \mu v+2 \zeta_{2} \mu \nu+2 \zeta_{1} & B_{3}=1 & C_{3}=0 \\
A_{4}=1 & &
\end{array}
$$

\section{DEFINITION OF THE PERFORMANCE CRITERIA}

In this paper the minimisation of the kinetic energy of the primary system and the maximisation of the power absorbed by the DVA are considered as tuning strategies of the DVA. Therefore, a performance index to be minimised is the integral of the kinetic energy of the primary mass calculated over the frequency-band $\pm \infty$ defined by:

$$
I_{\mathrm{k}}=\frac{m_{1} \mathrm{E}\left[\left|u_{1}\right|^{2}\right]}{2 S_{\mathrm{f}} \omega_{1} / k_{1}}
$$

where $E\left[\right.$ ] denotes the expectation value and $u_{1}$ is the velocity of the mass $m_{1}$. The performance index $I_{\mathrm{k}}$ represents the ratio of the kinetic energy of the primary system to the excitation force with a uniform spectrum density $S_{\mathrm{f}}(\omega)$. The unit of $S_{\mathrm{f}}(\omega)$ is $\mathrm{N}^{2} \mathrm{~s} / \mathrm{rad}$. The constant $S_{\mathrm{f}} \omega_{1} / k_{1}$ is introduced to ensure that the performance index is dimensionless. The mean squared value of the velocity of the primary mass can be written as:

$$
\mathrm{E}\left[\left|u_{1}\right|^{2}\right]=\frac{S_{\mathrm{f}} \omega_{1}}{m_{1} k_{1}} \int_{-\infty}^{+\infty}|\Gamma|^{2} \mathrm{~d} \lambda
$$

Substituting equation (6) in equation (5) yields:

$$
I_{\mathrm{k}}=\frac{1}{2} \int_{-\infty}^{+\infty}|\Gamma|^{2} \mathrm{~d} \lambda
$$

Thus, substituting equation (3) in (7) yields:

$$
I_{\mathrm{k}}=\frac{1}{2} \int_{-\infty}^{+\infty}\left|\frac{B_{0}+(\mathrm{j} \lambda) B_{1}+(\mathrm{j} \lambda)^{2} B_{2}+(\mathrm{j} \lambda)^{3} B_{3}}{A_{0}+(\mathrm{j} \lambda) A_{1}+(\mathrm{j} \lambda)^{2} A_{2}+(\mathrm{j} \lambda)^{3} A_{3}+(\mathrm{j} \lambda)^{4} A_{4}}\right|^{2} \mathrm{~d} \lambda
$$

Equation (8) can be integrated using the formula in reference [20] leading to:

$$
\begin{gathered}
I_{\mathrm{k}}=\frac{\pi\left(\mu v^{3} \zeta_{1}+\zeta_{2}+v^{2}\left(-2+(1+\mu) v^{2}+4 \zeta_{1}^{2}\right) \zeta_{2}+4\left(v+v^{3}\right) \zeta_{1} \zeta_{2}^{2}+4 v^{2} \zeta_{2}^{3}\right)}{4\left(\mu \nu^{3} \zeta_{1}^{2}+\zeta_{1}\left(1-2 v^{2}+(1+\mu)^{2} v^{4}+4 v^{2} \zeta_{1}^{2}\right) \zeta_{2}+\right.} \\
\left.v\left(\mu+4\left(1+(1+\mu) v^{2}\right) \zeta_{1}^{2}\right) \zeta_{2}^{2}+4(1+\mu) v^{2} \zeta_{1} \zeta_{2}^{3}\right)
\end{gathered}
$$

The power absorbed by the DVA is equal to that dissipated by the damper $c_{2}$, which can be written as:

$$
P_{2}(\omega)=\frac{1}{2} \operatorname{Re}\left\{f_{\mathrm{d}}^{*}(\mathrm{j} \omega)\left[u_{1}(\mathrm{j} \omega)-u_{2}(\mathrm{j} \omega)\right]\right\}
$$

Where $*$ denotes complex conjugate and the force $f_{\mathrm{d}}$ is the force produced by the damper given by:

$$
f_{\mathrm{d}}(\mathrm{j} \omega)=c_{2}\left(u_{1}(\mathrm{j} \omega)-u_{2}(\mathrm{j} \omega)\right)
$$


where $u_{2}$ is the velocity of the mass $m_{2}$. Substituting equation (11) in (10) the absorbed power by the DVA becomes:

$$
P_{2}(\omega)=\frac{1}{2} c_{2}\left|u_{1}(\mathrm{j} \omega)-u_{2}(\mathrm{j} \omega)\right|^{2}
$$

In this case the non-dimensional performance index is defined by:

$$
I_{\mathrm{p} 2}=\frac{c_{2} \mathrm{E}\left[\left|u_{1}-u_{2}\right|^{2}\right]}{4 \pi S_{\mathrm{f}} \omega_{1}^{2} / k_{1}}
$$

which represents the ratio of power absorbed by the DVA to that generated by excitation force with a spectrum density $S_{\mathrm{f}}$ acting on a damper of value $k_{1} /\left(\pi \omega_{1}^{2}\right)$. The constant $2 \pi S_{\mathrm{f}} \omega_{1}^{2} / k_{1}$ is introduced to ensure that the performance index is dimensionless. The mean squared value of the relative velocity times the mechanical damping $c_{2}$ can be expressed as follow:

$$
c_{2} \mathrm{E}\left[\left(u_{1}-u_{2}\right)^{2}\right]=\frac{S_{\mathrm{f}} \omega_{1}^{2}}{k_{1}} 2 \zeta_{2} \mu \nu \int_{-\infty}^{+\infty}|\Gamma-\Theta|^{2} \mathrm{~d} \lambda
$$

Thus the performance index becomes:

$$
I_{\mathrm{p} 2}=\frac{\zeta_{2} \mu \nu}{2 \pi} \int_{-\infty}^{+\infty}\left|\frac{D_{0}+(\mathrm{j} \lambda) D_{1}+(\mathrm{j} \lambda)^{2} D_{2}+(\mathrm{j} \lambda)^{3} D_{3}}{A_{0}+(\mathrm{j} \lambda) A_{1}+(\mathrm{j} \lambda)^{2} A_{2}+(\mathrm{j} \lambda)^{3} A_{3}+(\mathrm{j} \lambda)^{4} A_{4}}\right|^{2} \mathrm{~d} \lambda
$$

where

$$
\begin{aligned}
& D_{0}=B_{0}-C_{0}=0 \\
& D_{1}=B_{1}-C_{1}=0 \\
& D_{2}=B_{2}-C_{2}=0 \\
& D_{3}=B_{3}-C_{3}=\mu
\end{aligned}
$$

The integral over the frequency band between $\pm \infty$ of equation (15) can be calculated using the expression given in reference [20], leading to:

$$
\begin{aligned}
I_{p 2}= & \frac{\mu \nu \zeta_{2}\left(\zeta_{2}+v \zeta_{1}\left((1+\mu) v^{2}+4 \zeta_{2}\left(\nu \zeta_{1}+\zeta_{2}\right)\right)\right)}{4\left(\mu v^{3} \zeta_{1}^{2}+\zeta_{1}\left(1-2 v^{2}+(1+\mu)^{2} v^{4}+4 v^{2} \zeta_{1}^{2}\right) \zeta_{2}+\right.} \\
& \left.v\left(\mu+4\left(1+(1+\mu) v^{2}\right) \zeta_{1}^{2}\right) \zeta_{2}^{2}+4(1+\mu) v^{2} \zeta_{1} \zeta_{2}^{3}\right)
\end{aligned}
$$

Although the denominators are the same in equations (9) and (16), the dependence of their numerators on $\zeta_{2}$ and $v$ is clearly different.

\section{MINIMISATION OF KINETIC ENERGY AND MAXIMISATION POWER ABSORBED}

In order to minimise the total kinetic energy of the primary mass $m_{1}$, the following conditions have to be satisfied:

$$
\left\{\begin{array}{l}
\frac{\partial I_{\mathrm{k}}}{\partial \zeta_{2}}=0 \\
\frac{\partial I_{\mathrm{k}}}{\partial v}=0
\end{array}\right.
$$


while to maximise the total power absorbed by the DVA the following conditions have to be satisfy:

$$
\left\{\begin{array}{l}
\frac{\partial I_{\mathrm{p} 2}}{\partial \zeta_{2}}=0 \\
\frac{\partial I_{\mathrm{p} 2}}{\partial v}=0
\end{array}\right.
$$

Differentiating the performance index $I_{\mathrm{k}}$ expressed in equation (9) with respect to $\zeta_{2}$ and $v$, and setting these equal to zero, yields a system of two equations:

$$
\begin{gathered}
-\mu v\left[\left(\zeta_{1}^{2} \mu(\mu+1) v^{6}+\zeta_{2}^{2}\left(4 \zeta_{1}^{2} v^{2}\left(4 \zeta_{1}^{2} v^{2}+(2 \mu-3) v^{2}+2\right)+(\mu+1) v^{4}-2 v^{2}+1\right)\right.\right. \\
\left.\left.+2 \zeta_{1} \zeta_{2} \mu v^{3}\left(4 \zeta_{1}^{2} v^{2}+1\right)+4\left(4 \zeta_{1}^{2}-1\right) \zeta_{2}^{4} v^{2}+8 \zeta_{1} \zeta_{2}^{3} v\left(4 \zeta_{1}^{2} v^{2}-2 v^{2}+1\right)\right)\right]=0 \\
-\zeta_{2} \mu\left[\left(\zeta_{1}^{2} \mu(\mu+1) v^{6}+\zeta_{2}^{2}\left(4 \zeta_{1}^{2} v^{2}\left(4 \zeta_{1}^{2} v^{2}+(2 \mu+1) v^{2}+2\right)-3(\mu+1) v^{4}+2 v^{2}+1\right)\right.\right. \\
+2 \zeta_{1} \zeta_{2} v^{3}\left(4 \zeta_{1}^{2}(\mu+1) v^{2}-2(\mu+1) v^{2}+\mu+2\right)+4\left(4 \zeta_{1}^{2}-1\right) \zeta_{2}^{4} v^{2} \\
\left.\left.+8 \zeta_{1} \zeta_{2}^{3} v\left(4 \zeta_{1}^{2} v^{2}-v^{2}+1\right)\right)\right]=0
\end{gathered}
$$

Following the same procedure, the partial derivates of the performance index $I_{\mathrm{p} 2}$ expressed in equation (16) are given by:

$$
\begin{gathered}
\zeta_{1} \mu v\left[\zeta_{1}^{2} \mu(\mu+1) v^{6}+\zeta_{2}^{2}\left(4 \zeta_{1}^{2} v^{2}\left(4 \zeta_{1}^{2} v^{2}+(2 \mu-3) v^{2}+2\right)+(\mu+1) v^{4}-2 v^{2}+1\right)\right. \\
\left.+2 \zeta_{1} \zeta_{2} \mu v^{3}\left(4 \zeta_{1}^{2} v^{2}+1\right)+4\left(4 \zeta_{1}^{2}-1\right) \zeta_{2}^{4} v^{2}+8 \zeta_{1} \zeta_{2}^{3} v\left(4 \zeta_{1}^{2} v^{2}-2 v^{2}+1\right)\right]=0 \\
\zeta_{1} \mu v\left[\left(\zeta_{1}^{2} \mu(\mu+1) v^{6}+\zeta_{2}^{2}\left(4 \zeta_{1}^{2} v^{2}\left(4 \zeta_{1}^{2} v^{2}+(2 \mu+1) v^{2}+2\right)-3(\mu+1) v^{4}+2 v^{2}+1\right)\right.\right. \\
+2 \zeta_{1} \zeta_{2} v^{3}\left(4 \zeta_{1}^{2}(\mu+1) v^{2}-2(\mu+1) v^{2}+\mu+2\right)+4\left(4 \zeta_{1}^{2}-1\right) \zeta_{2}^{4} v^{2} \\
\left.\left.+8 \zeta_{1} \zeta_{2}^{3} v\left(4 \zeta_{1}^{2} v^{2}-v^{2}+1\right)\right)\right]=0
\end{gathered}
$$

Simultaneous equation (19) and (20) are both satisfied for $\zeta_{2}=0$ and $v=0$ corresponding to maximising $I_{\mathrm{k}}$ and $I_{\mathrm{p} 2}$. The other solutions can be found setting to zero the terms in squared brackets. If $\zeta_{1}=0$ equations (20) are satisfied for any value of $\zeta_{2}$ and $v$ indicating that the power absorbed by the DVA is constant. Solving equations (19) the optimal values of $\zeta_{2}$ and $v$ are given by:

$$
\begin{gathered}
\zeta_{\mathrm{opt}}=\frac{\sqrt{\mu}}{2} \\
v_{\mathrm{opt}}=\frac{1}{\sqrt{1+\mu}}
\end{gathered}
$$

as also found in reference [18]. The term in square brackets in equation (19a) is equal to the term in square brackets in equation (20a) and the term in square brackets in equation (19b) is equal to the term in square brackets in equation (20b). Provided that $\mu$ or $\zeta_{1}$ are not identically zero, then these terms in square brackets must both be zero, and so the minimum of the kinetic energy and the maximum of the power absorbed must both correspond to the same optimum values of $\zeta_{2}$ and $v$. 
Figure 2 shows the power spectral density of the velocity of the primary mass in dimensionless form for five different strategies of tuning the DVA. The area under the curve is minimised when the minimisation of the kinetic energy is implemented. The highest response is obtained when the stability maximisation criterion is implemented because this criterion is not designed to improve the steady state response but only the transient response of the system.

\section{POWER INPUT}

From the energy balance, in steady state conditions, the power input into the system is equal to the power dissipated by damper 1 plus the power dissipated by damper 2 . The power dissipated by damper 1 is given by:

$$
P_{1}(\omega)=\frac{1}{2} c_{1}\left|u_{1}(\mathrm{j} \omega)\right|^{2}
$$

In order to obtain a non-dimensional formulation, as already done in previous sections, a nondimensional index relative to $P_{1}(\omega)$ can be defined by:

$$
I_{\mathrm{p} 1}=\frac{c_{1} E\left[\left|u_{1}\right|^{2}\right]}{4 \pi S_{\mathrm{f}} \omega_{1}^{2} / k_{1}}
$$

Therefore the sum of $I_{\mathrm{p} 1}$ and $I_{\mathrm{p} 2}$ gives the non-dimensional power input into the system. The mean squared value of the velocity of mass $m_{1}$ times the mechanical damping $c_{1}$ can be expressed as follow:

$$
c_{1} E\left[\left|u_{1}\right|^{2}\right]=\frac{S_{\mathrm{f}} \omega_{1}^{2}}{c_{1}} 2 \zeta_{1} \int_{-\infty}^{+\infty}|\Gamma|^{2} d \lambda
$$

Substituting equation (24) in (23), the performance index becomes:

$$
I_{\mathrm{p} 1}=\frac{\zeta_{1}}{2 \pi} \int_{-\infty}^{+\infty}\left|\frac{B_{0}+(j \lambda) B_{1}+(j \lambda)^{2} B_{2}+(j \lambda)^{3} B_{3}}{A_{0}+(j \lambda) A_{1}+(j \lambda)^{2} A_{2}+(j \lambda)^{3} A_{3}+(j \lambda)^{4} A_{4}}\right|^{2} d \lambda
$$

Integrating equation (25), $I_{\mathrm{p} 1}$ can be written as:

$$
\begin{gathered}
I_{\mathrm{p} 1}=\frac{\zeta_{1}\left(\mu \nu^{3} \zeta_{1}+\zeta_{2}+v^{2}\left(-2+(1+\mu) v^{2}+4 \zeta_{1}^{2}\right) \zeta_{2}+4\left(v+v^{3}\right) \zeta_{1} \zeta_{2}^{2}+4 v^{2} \zeta_{2}^{3}\right)}{4\left(\mu \nu^{3} \zeta_{1}^{2}+\zeta_{1}\left(1-2 v^{2}+(1+\mu)^{2} v^{4}+4 v^{2} \zeta_{1}^{2}\right) \zeta_{2}+\right.} \\
\left.v\left(\mu+4\left(1+(1+\mu) v^{2}\right) \zeta_{1}^{2}\right) \zeta_{2}^{2}+4(1+\mu) v^{2} \zeta_{1} \zeta_{2}^{3}\right)
\end{gathered}
$$

The power input into the system can be calculated as the sum of equation (16) and (26). Summing the two expressions the result is $1 / 4$, which, multiplied by the constant $2 \pi S_{\mathrm{f}} \omega_{1}^{2} /$ $k_{1}$ used to make the performance indices $I_{\mathrm{p} 1}$ and $I_{\mathrm{p} 2}$ non-dimensional leads to:

$$
P_{\text {in }}=\frac{\pi}{2 m_{1}}
$$

where $P_{\text {in }}$ is the power input into the system. Equation (27) indicates that the power input into the systemonly depends on the mass $m_{1}$. This means that the derivates of $I_{\mathrm{p} 1}$ are equal to the derivates of $I_{\mathrm{p} 2}$. Because the kinetic energy of the primary system is proportional to the power 
dissipated by the damper 1 its derivates are proportional to the derivates of $I_{\mathrm{p} 2}$ and therefore the minimisation of the kinetic energy corresponds to the maximisation of the power absorbed by the DVA as shown in the previous section.

\section{CONCLUSIONS}

It is shown that for a damped host structure, the ratios of natural frequencies and absorber damping that maximise the power dissipation in the absorber are the same as those that minimise the kinetic energy of the host structure. Therefore the effect of maximising the absorbed power of the DVA is to minimise the kinetic energy of the host structure, modeled as a single degree of freedom. This may provide a method of self-tuning such a dynamic vibration absorber. If the power dissipation in the absorber could be measured and the disturbance was stationary, a tuning strategy might be used that is similar to that used for feedback controllers in references $[21,22]$.

One method of measuring the power dissipation within the absorber may be to measure its temperature. Alternatively, if the tuned vibration absorber was implemented with an efficient inertial electromagnetic actuator, most of the mechanical power dissipation could then be arranged to be the electrical power generated in a tuneable shunting impedance. It may be possible to use this power both to tune the absorber, by adjusting this electrical impedance, and also, using energy harvesting techniques, to power the electronic system used to implement the self-tuning.

\section{APPENDIX A}

In this appendix the analytical expression of the mobility functions of the system shown in Figure 1 are defined. The equation of motion of the system shown in Figure 1 can be written in a matrix form as:

$$
\mathbf{M} \ddot{\mathbf{x}}(t)+\mathbf{C} \dot{\mathbf{x}}(t)+\mathbf{K x}(t)=\boldsymbol{f}(t)
$$

Where $\mathbf{M}$ is the mass matrix, $\mathbf{K}$ is the stiffness matrix and $\mathbf{C}$ is the damping matrix given by:

$$
\mathbf{M}=\left[\begin{array}{cc}
m_{1} & 0 \\
0 & m_{2}
\end{array}\right], \mathbf{K}=\left[\begin{array}{cc}
k_{1}+k_{2} & -k_{2} \\
-k_{2} & k_{2}
\end{array}\right], \mathbf{C}=\left[\begin{array}{cc}
c_{1}+c_{2} & -c_{2} \\
-c_{2} & c_{2}
\end{array}\right],
$$

$\mathbf{x}(t)=\left[\begin{array}{ll}x_{1}(t) & x_{2}(t)\end{array}\right]^{T}$ is the column vector containing the displacements of the two masses $x_{1}$ and $x_{2}$ and $\mathbf{f}(t)=\left[\begin{array}{lll}f_{\mathrm{p}}(t) & 0\end{array}\right]^{T}$ is the column vector of primary excitation. 
Assuming the excitation to be harmonic for the time being and expressing the force and the steady-state response in exponential form, equation (A 1) becomes:

$$
\mathbf{S}(\mathrm{j} \omega) \mathbf{x}(\mathrm{j} \omega)=\mathbf{f}(\mathrm{j} \omega)
$$

where

$$
\mathbf{S}(\mathrm{j} \omega)=-\omega^{2} \mathbf{M}+\mathrm{j} \omega \mathbf{C}+\mathbf{K}
$$

is the dynamic stiffness matrix. The solution of equation (A 3) can be obtained as:

$$
\mathbf{x}(\mathrm{j} \omega)=\mathbf{S}^{-1}(\mathrm{j} \omega) \mathbf{f}(\mathrm{j} \omega)
$$

Integrating equation (A 5) to obtain the velocities yields:

$$
\mathbf{v}(\mathrm{j} \omega)=\mathbf{Y}(\mathrm{j} \omega) \mathbf{f}(\mathrm{j} \omega)
$$

wherev $(j \omega)=j \omega \mathbf{x}(j \omega)$ and $\mathbf{Y}(j \omega)=j \omega \mathbf{S}^{-1}(j \omega)$ is the mobility matrix. Using the expression of $\mathbf{M}, \mathbf{K}$ and $\mathbf{C}$ of equation (A 2) the velocity per unit input force of the two masses is given by:

$$
\begin{aligned}
& Y_{11}(j \omega)=\frac{u_{1}}{f_{\mathrm{p}}}= \\
& =\frac{\mathrm{j} k_{2} \omega-\mathrm{c}_{2} \omega^{2}-\mathrm{j} m_{2} \omega^{3}}{k_{1} k_{2}+\mathrm{jc}_{2} k_{1} \omega+\mathrm{jc}_{1} k_{2} \omega-\mathrm{c}_{1} \mathrm{c}_{2} \omega^{2}-k_{2} m_{1} \omega^{2}-k_{1} m_{2} \omega^{2}-k_{2} m_{2} \omega^{2}-\mathrm{jc}_{2} m_{1} \omega^{3}-} \\
& Y_{12}(j \omega)=\frac{u_{2}}{\mathrm{jc}_{1} m_{2} \omega^{3}-\mathrm{jc} \mathrm{c}_{2} m_{2} \omega^{3}+m_{1} m_{2} \omega^{4}} \\
& =\frac{\mathrm{j} k_{2} \omega-\mathrm{c}_{2} \omega^{2}}{k_{1} k_{2}+\mathrm{jc}_{2} k_{1} \omega+\mathrm{jc}_{1} k_{2} \omega-\mathrm{c}_{1} \mathrm{c}_{2} \omega^{2}-k_{2} m_{1} \omega^{2}-k_{1} m_{2} \omega^{2}-k_{2} m_{2} \omega^{2}-\mathrm{jc}_{2} m_{1} \omega^{3}-} \\
& \mathrm{jc}_{1} m_{2} \omega^{3}-\mathrm{jc}_{2} m_{2} \omega^{3}+m_{1} m_{2} \omega^{4}
\end{aligned}
$$

\section{REFERENCES}

[1] J.P. Den Hartog, Mechanical Vibrations, 4th ed., McGraw-Hill, New York, 1956.

[2] H. Frahm, Device for damping vibrations of bodies, in: U. Patent (Ed.), 1911.

[3] P. Dallard, A.J. Fitzpatrick, A. Flint, S.L. Bourva, A. Low, R.M. Ridsdill-Smith, M. Willford, The London Millennium Footbridge, Structural Engineer, 79 (2001) 17-33.

[4] J.Q. Sun, M.R. Jolly, M.A. Norris, Passive, Adaptive and Active Tuned Vibration Absorbers---A Survey, Journal of Vibration and Acoustics, 117 (1995) 234-242.

[5] B.F. Spencer, Jr., M.K. Sain, Controlling buildings: a new frontier in feedback, Control Systems, IEEE, 17 (1997) 19-35.

[6] A.H. von Flotow, Beard, A., and Bailey, D., Adaptive tuned vibration absorbers: tuning laws, tracking agility, sizing and physical implementations, in: Noise-Con 94, Fort Lauderdale, FL, 1994, pp. 437-454.

[7] A.H. von Flotow, M. Mercadal, K.B. Scribner, T. Mixon, C. Roeseler, Adaptively tuned vibration absorber for reduction of aircraft cabin noise, in, US Patent 5,873,559, 1999.

[8] C.R. Fuller, J.P. Maillard, M. Mercadal, A.H. von Flotow, Control of aircraft interior noise using globally detuned vibration absorber, Journal of Sound and Vibration, 203 (1997) 745-761. 
[9] P. Bonello, M.J. Brennan, S.J. Elliott, J.F.V. Vincent, G. Jeronimidis, Designs for an adaptive tuned vibration absorber with variable shape stiffness element, Proceedings of the Royal Society A: Mathematical, Physical and Engineering Science, 461 (2005) 3955-3976.

[10] E. Rustighi, M.J. Brennan, B.R. Mace, A shape memory alloy adaptive tuned vibration absorber: design and implementation, Smart Materials and Structures, 14 (2005).

[11] T. Asami, O. Nishihara, A.M. Baz, Analytical solutions to $\mathrm{H} \infty$ and $\mathrm{H} 2$ optimization of dynamic vibration absorbers attached to damped linear systems, Journal of vibration and acoustics 124 (2002) 284-295.

[12] J. Ormondroyd, J.P. DenHartog, The theory of the Dynamic Vbration Absorber, Journal of Applied Mechanics, 50 (1928) 9-22.

[13] O. Nishihara, T. Asami, Closed-Form Solutions to the Exact Optimizations of Dynamic Vibration Absorbers (Minimizations of the Maximum Amplitude Magnification Factors), Journal of Vibration and Acoustics, 124 (2002) 576-582.

[14] S.H. Crandall, W.D. Mark, Random vibration in mechanical systems, Academic Press, 1963.

[15] H. Yamaguchi, Damping of Transient vibration by a Dynamic Absorber, Transactions of the Japan Society of Mechanical Engineers, Ser. C, 54 (1988) 561-568.

[16] D.W. Miller, E.F. Crawley, B.A. Wards, Inertial actuator design for maximum passive and active energy dissipation in flexible space structures, in: Structures, Structural Dynamics, and Materials Conference American Institute of Aeronautics and Astronautics, Orlando FL, April 1985, pp. 536-544.

[17] S. Krenk, Frequency Analysis of the Tuned Mass Damper, Journal of Applied Mechanics, 72 (2005) 936-942.

[18] G.B. Warburton, Optimum absorber parameters for various combinations of response and excitation parameters, Journal of earthquake engineering and structural dynamics 10 (1982) 381-401.

[19] Y. Iwata, On the construction of the dynamic vibration absorber, Japanese Society of Mechanical Engineering, 820 (1982) 150-152.

[20] D.E. Newland, An Introduction to Random Vibrations, Spectral \& Wavelet Analysis, Third Edition ed., Dover Publications, Inc, Mineola, New York, 1975.

[21] M. Zilletti, S.J. Elliott, P. Gardonio, Self-tuning control systems of decentralised velocity feedback, Journal of Sound and Vibration, 329 (2010) 2738-2750.

[22] M. Zilletti, S.J. Elliott, P. Gardonio, E. Rustighi, Experimental implementation of a selftuning control system for decentralised velocity feedback, Journal of Sound and Vibration, 331 (2012) 1-14.

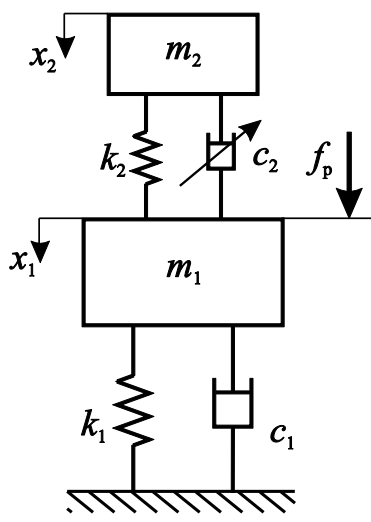

Figure 1: Scheme of the SDOF system with the DVA 


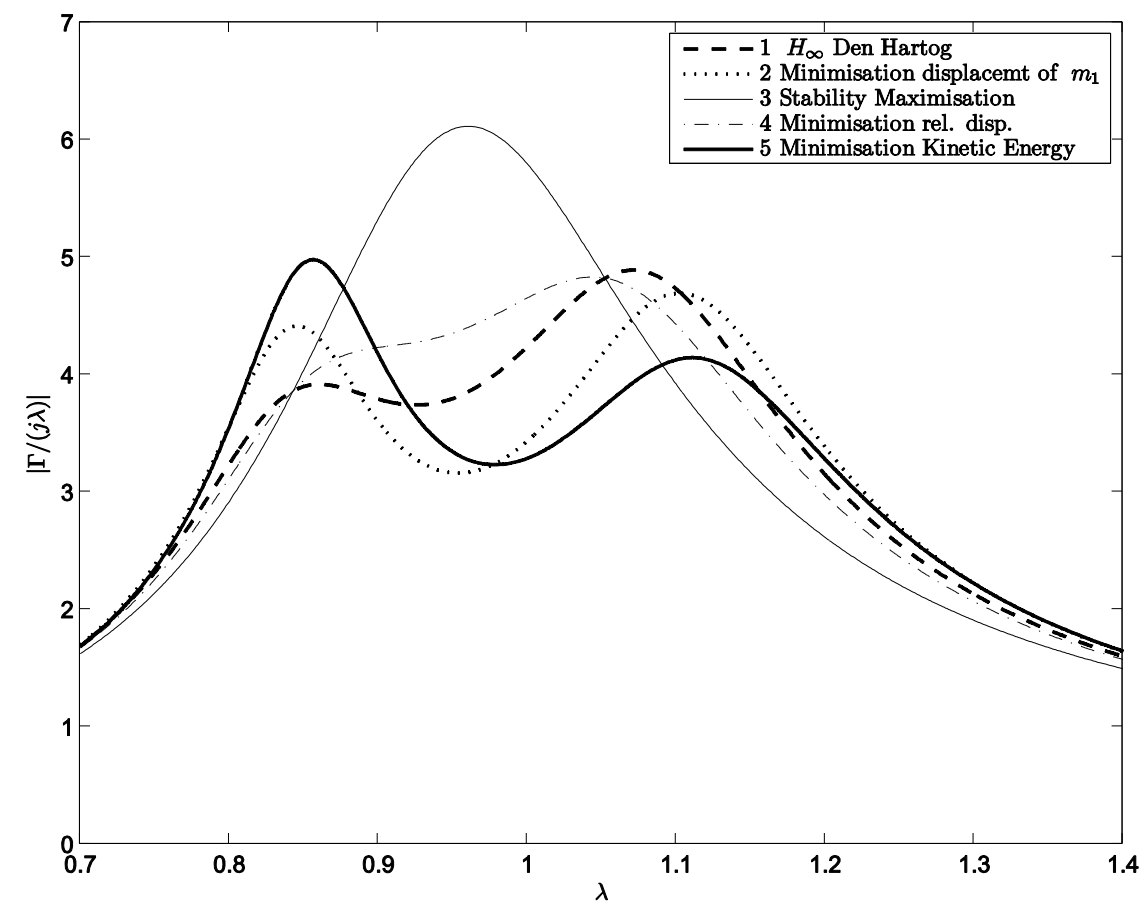

Figure 2: Optimal power spectral density of the dimensionless velocity of the primary system when the five different criteria of Table 1 are implemented to tune the DVA $\left(\zeta_{1}=0, \mu=0.1\right)$ 